\title{
Controle Automático de Volume em Tempo Real Utilizando Inferência Fuzzy em um Sistema Embarcado
}

\author{
A. T. ESCOTTÁ ${ }^{1}$ e W. BECCARO ${ }^{2 *}$ \\ Recebido em 4 de fevereiro de 2019 / Aceito em 6 de outubro de 2020
}

\begin{abstract}
RESUMO. Controle Automático de Volume (CAV) é uma tecnologia que ajusta automaticamente a intensidade sonora de um sinal na presença de ruído. Diferentes técnicas para realizar o CAV foram relatadas na literatura, contudo poucos estudos utilizam algoritmos de inteligência computacional. A lógica Fuzzy é uma técnica que suporta modos de raciocínio com o princípio da incerteza, sendo capaz de aproximar o pensamento humano e aspectos psicoacústicos em aplicações de áudio. Este artigo apresenta um CAV utilizando sistema de inferência Fuzzy, que permite o controle de volume em tempo real de um sinal de áudio, mantendo a capacidade de discriminação de um som mesmo em um ambiente ruidoso. Os resultados da caracterização do algoritmo Fuzzy embarcado em um microprocessador ARM Cortex M4, demonstram o ajuste automático e rápido do volume de áudio de saída através de regras Fuzzy.
\end{abstract}

Palavras-chave: lógica fuzzy, sistemas multimídias, sistemas embarcados, Controle Automático de Volume.

\section{INTRODUÇÃO}

O Controle Automático de Volume (CAV) $[9,12]$ é uma técnica utilizada para compensar ruídos e interferências externas, garantindo inteligibilidade sonora [9]. O CAV pode ser utilizado em diferentes aplicações, como por exemplo: telefones celulares, televisores [13], sistemas de áudio, sistemas de telepresença e videoconferência [14, 19,35,38], áudio automotivo [1], entre outros $[2,22,33]$. Tais sistemas são capazes de monitorar o nível de ruído do ambiente e realizar o ajuste de compensação de volume. Esse controle é realizado através do aumento da intensidade sonora do emissor do áudio de interesse, garantindo a inteligibilidade do sinal em um limiar constante e seguro para o usuário.

Os projetos e as análises de CAV têm sido explorados desde a década de 1920, inicialmente concebidos por circuitos elétricos realimentados. Com os avanços tecnológicos, tornaram-se importantes os métodos que podem ser implementados em hardwares e softwares. Wheeler [37]

\footnotetext{
*Corresponding author: Wesley Beccaro - E-mail: wesley@lme.usp.br

${ }^{1}$ Departamento de Engenharia de Sistemas Eletrônicos da Escola Politécnica da Universidade de São Paulo, São Paulo, SP, Brasil. - E-mail: escotta.alvaro@ gmail.com https://orcid.org/0000-0002-5128-3966

${ }^{2}$ Departamento de Engenharia de Sistemas Eletrônicos da Escola Politécnica da Universidade de São Paulo, São Paulo, SP, Brasil. - E-mail: wesley@lme.usp.br https://orcid.org/0000-0001-6599-2344
} 
propôs um circuito elétrico capaz de regular automaticamente a amplificação de rádiofrequência (RF), de modo a fornecer uma tensão quase constante no detector de RF, resultando em uma resposta uniforme no alto falante. Griffith [12] aponta os circuitos mais bem-sucedidos para um CAV, caracterizado por um método de absorção ou desvio, no qual a resistência do emissor de um amplificador de base comum é o elemento variável do circuito.

Diversos trabalhos apresentam soluções digitais e modernas para o CAV utilizando aplicações e algoritmos específicos $[9,18]$. Grande parte utiliza análise espectral e estimação da intensidade sonora $[9,15,18,19]$, o que possibilita calcular deterministicamente o valor do volume da saída de áudio. Dentre estes trabalhos, Han et al. [13] propõe um CAV a fim de compensar a diferença de volume entres canais de televisão provocadas pela taxa de modulação e demodulação do sinal. O sistema apresenta uma proposta de estimação de potência e um algoritmo para compensação de volume levando em consideração a sensibilidade auditiva humana. A estimativa de potência é baseada em um algoritmo para cálculo dos valores máximo, mínimo, e da potência eficaz do sinal. Felber [9] aborda um CAV para todos os dispositivos que contém ao menos um microfone e estão expostos a ambientes ruidosos. O método utiliza algoritmos embarcados com a capacidade de análise do ruído de fundo em tempo real, realizando a compensação das flutuações no domínio do tempo e frequência garantindo a inteligibilidade constante da fala. Kabir et al. [17] apresenta um CAV para sistemas de TV e rádio. O método proposto baseia-se em correlação entre o sinal reproduzido e o sinal de saída que consiste no sinal reproduzido acrescido do ruído externo. Utilizando esta estratégia, o algoritmo ajusta o volume considerado aspectos de atraso e forma de onda. Takahashi et al. [35] tratam da interface de usuário de um robô para aplicações de telepresença que contempla um CAV de acordo com a distância entre o robô e o usuário e o nível de ruído ambiente.

Diversas patentes [2, 6, 19, 21, 22, 33] foram propostas apresentando modos variados de implementação de um CAV a fim de compensar variações de ruídos ambientes. Com base na relação sinal-ruído, os sistemas determinam o nível de volume de acordo com condição de entrada imposta de modo que o usuário ouça o áudio confortavelmente.

No tocante a algoritmos de inteligência computacional, Yang et al. [38] propõe um sistema com CAV em malha aberta com recurso de Deep Learning. O sistema possui blocos de detecção de conteúdo de sinal e silêncio, estimativa do nível de sinal, além do aprendizado do ganho. Cada um dos recursos é treinado com base em um modelo de aprendizado de máquina a partir de métricas estatísticas. Al-Jarrah et al. [1] apresenta resultados de simulação de uma aplicação CAV em ambiente automotivo para compensação de ruídos ambientes utilizando conceitos Fuzzy. Contudo, até onde os autores conhecem, não há reportado na literatura aplicações embarcadas em tempo real de lógica Fuzzy empregadas em sistemas de CAV.

Este trabalho apresenta a proposta, o desenvolvimento e a caracterização de um CAV em tempo real com base no nível de ruído externo baseado na teoria de conjuntos Fuzzy [29,40,41] para o modelamento da tomada de decisão parametrizada em conformidade com o raciocínio e características humanas. $\mathrm{O}$ artigo traz a novidade da prova de conceito da implementação de um CAV de tempo real baseado em regras Fuzzy embarcado em uma plataforma da família ARM Cortex 
M4 para compensar ruídos na faixa de $21 \mathrm{~dB}$ até $72 \mathrm{~dB}$. Os resultados podem ser generalizados em diversas aplicações embarcadas, como é o caso de smartphones, tablets, equipamentos de áudio, televisores, entre outros. Além disso, através do controle baseado em Fuzzy, podem-se contemplar questões relacionadas à psicoacústica, bem como criar mecanismos que permitam adicionar a experiência humana à determinação dos valores relativos ao aumento e decréscimo da intensidade do som [26]. Para tanto são utilizadas técnicas de processamento digital de sinais e modulação por código de pulso, PCM (Pulse-Code Modulation). Este trabalho também apresenta a implementação de uma biblioteca escrita em linguagem ANSI C para desenvolvimento de sistemas baseados em lógica Fuzzy em plataformas de microcontroladores da família ARM Cortex M. A biblioteca possibilita, a partir dos recursos limitados de microcontroladores e processadores digital de sinais de baixo custo, a execução das lógicas relacionados aos processos de fuzzyficação, inferência Fuzzy e defuzzyficação de maneira eficiente, possibilitando o desenvolvimento de sistemas de controle inteligente em tempo real.

O artigo está estruturado como se segue. A Seção 2 apresenta os conceitos de lógica Fuzzy. Na Seção 3, discutem-se os materiais e métodos utilizados para o projeto do CAV. A Seção 4 apresenta as caracterizações e testes funcionais realizados, e as conclusões são detalhadas na Seção 5.

\section{CONCEITOS DE LÓGICA FUZZY}

A lógica Fuzzy, proposta inicialmente em 1965 [40], tem sido aplicada em diversas áreas [3,4,5, 11]. O conceito fundamental da lógica Fuzzy é flexibilizar a pertinência dos elementos através do conceito de graus de pertinência [16,34], permitindo assim modelar a incerteza e a subjetividade de sistemas complexos.

Os conjuntos Fuzzy têm como principal característica não apresentar fronteiras bem definidas, diferente da lógica clássica que utiliza distinções estritamente definidas para tratamento de classes de objetos. Os conjuntos Fuzzy apresentam nomes ou rótulos denominados de variáveis linguísticas, cuja principal função é fornecer uma maneira sistemática de aproximação de fenômenos complexos, subjetivos, ou mal definidos [16]. Além disso, são representados por funções de pertinência, no qual os critérios de seleção dependem do problema a ser modelado e da capacidade computacional em relação ao processamento das informações. Comumente, as funções de pertinência mais usuais são as lineares (triangulares e trapezoidais), as gaussianas e as quadráticas.

Um sistema baseado na lógica Fuzzy é capaz de produzir saídas numéricas a partir de entradas Fuzzy com base em uma metodologia que articula ações através de um conjunto de regras da forma "Se-Então". Com isso, é possível a implementação de um algoritmo Fuzzy, tipicamente composto pelos módulos funcionais de fuzzyficação, inferência Fuzzy e defuzzyficação. Um esquema sequencial dos módulos que compõe o sistema Fuzzy está apresentado na Figura 1. 


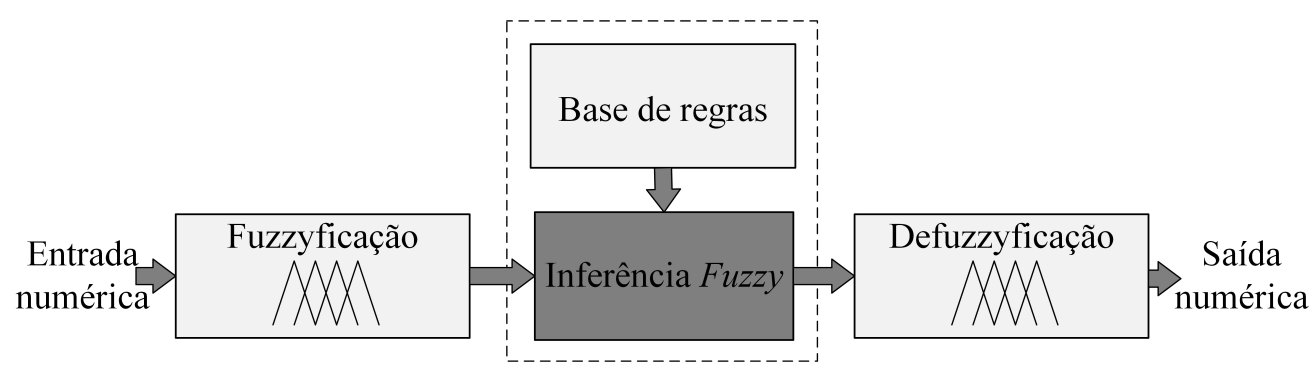

Figura 1: Esquema sequencial dos blocos de um sistema baseado em lógica Fuzzy.

O primeiro bloco, relativo à fuzzyficação, corresponde ao processo de mapeamento da entrada numérica do sistema em graus de pertinência, ou seja, a conversão de um valor numérico em um conjunto Fuzzy [16,31]. Assim, cada entrada do sistema é associada à uma função de pertinência. O mapeamento de entrada/saída é realizado através de processos de inferência Fuzzy. Para isso, são definidas regras estabelecidas por especialistas. Com base nessas regras a inferência Fuzzy interpreta os valores de entrada Fuzzy assimilando-os a um valor de saída Fuzzy. Existem vários modelos de inferência, sendo que o método de Mamdani é amplamente utilizado [31]. O método de Mamdani agrupa as diferentes entradas e as regras de inferência com o objetivo de agregar a saída para as $n$ regras estabelecidas [24,25]. Na Figura 2 são exemplificadas duas regras que mapeiam entradas, que podem ser números reais ou $n$-upla de números reais, ambas entradas também conhecidas como crisp. Embora existam diferentes mecanismos de composição das relações Fuzzy, são amplamente difundidas as composições baseadas em max-min. Nesses casos, a superfície dos conjuntos Fuzzy de saída é obtida utilizando o menor grau de pertinência dos conjuntos de fuzzyficação e, posteriormente, considerando o máximo das intersecções entre termos linguísticos dos conjuntos de pertinência da deffuzyficação, conforme equacionado em (2.1) e descrito de modo ilustrativo na Figura 2.

$$
\mu_{C_{n}}(z)=\max _{n}\left\{\min \left[\mu_{A_{n}}(x), \mu_{B_{n}}(y)\right]\right\}
$$

no qual $A, B$ e $C$ são variáveis linguísticas definidas por conjuntos Fuzzy no universo do discurso $X, Y$ e $Z$, respectivamente, e $\mu_{C_{n}}, \mu_{A_{n}}$, e $\mu_{B_{n}}$ correspondem às funções de pertinência da saída $z$, e das entradas $x$ e $y$, respectivamente.

Após o processo de inferência, a defuzzyficação converte a saída Fuzzy e um número real. Existem muitas técnicas de obtenção do valor numérico de saída, entre elas o método do centroide, que calcula o centro de gravidade da distribuição de possibilidades de saída do sistema Fuzzy, tal como descrito em (2.2) para casos de variáveis discretas. A vantagem do uso do método do centroide é considerar toda a forma do conjunto Fuzzy de saída para se obter o resultado numérico defuzzyficado.

$$
d_{\text {centroide }}=\frac{\sum_{k=1}^{N} \mu\left(z_{k}\right) z_{k}}{\sum_{k=1}^{N} \mu\left(z_{k}\right)}
$$




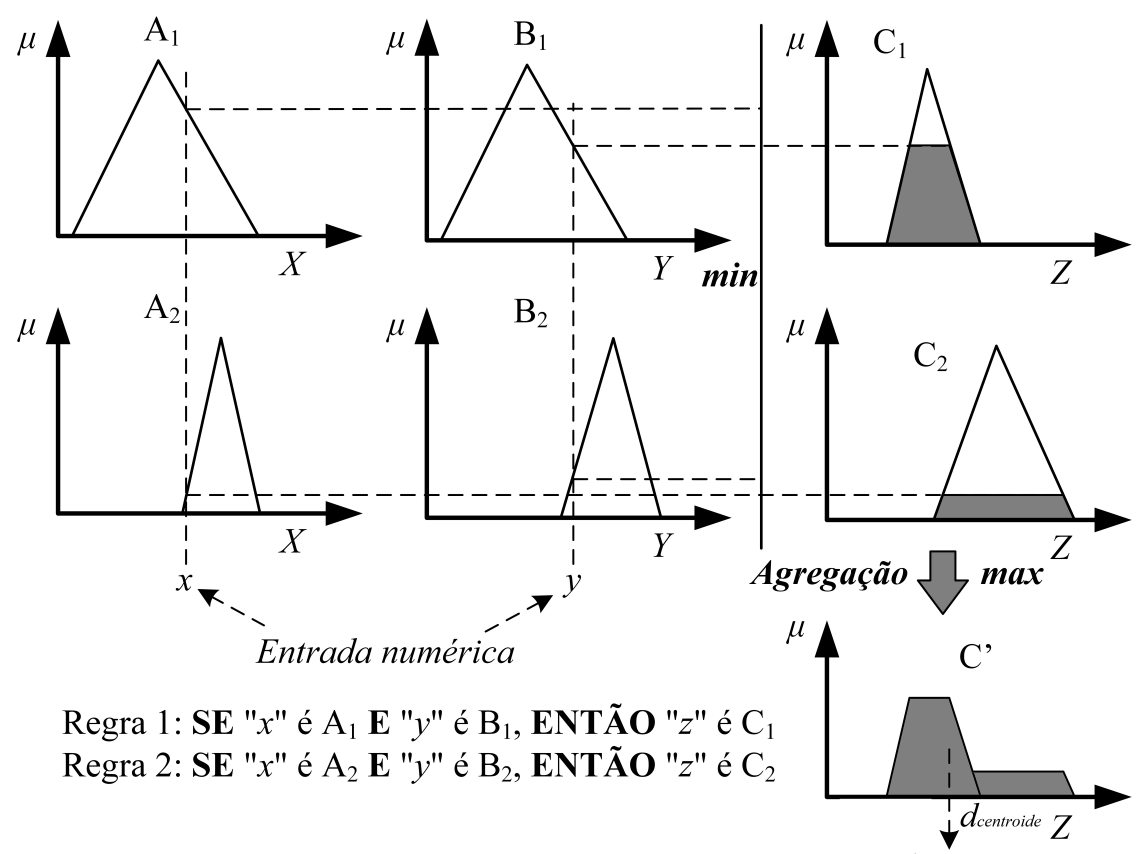

Saída numérica

Figura 2: Etapas da lógica Fuzzy: fuzzyficação das entradas numéricas $x$ e $y$, exemplificação de duas regras baseadas na inferência Mamdani, mecanismo de composição max-min, defuzzyficação e saída numérica obtida através do método do centroide [17].

no qual $z_{k}$ é a variável linguística, $\mu\left(z_{k}\right)$ é o grau de pertinência da variável linguística, $k$ é o número total de regras e $d_{\text {centroide }}$ corresponde à saída numérica do sistema Fuzzy.

Existem muitas abordagens referentes à utilização de lógica Fuzzy tanto em processos de tempo real, como em análises off-line. Uma das tendências, com o crescimento da indústria de embarcados, é o uso destes dispositivos na monitoração e controle de diversos sistemas e processos [8,27, 32]. A utilização de lógica Fuzzy embarcada, como apresentado neste trabalho, possibilita aumentar o desempenho, a confiabilidade e a produtividade no controle de sistemas complexos em tempo real, além de diminuir o custo de mercado, o tamanho do produto e a duração do desenvolvimento do projeto.

\section{MATERIAIS E MÉTODOS}

Esta seção apresenta os principais elementos que compõem a estrutura do CAV, além de descrever os métodos de implementação dos algoritmos de controle e da caracterização do sistema. O CAV proposto atua em embarcado em um processador digital de sinais, em tempo real e isoladamente no controle do volume do áudio de acordo com o nível de ruído captado. 
As condições descritas neste trabalho consideram um usuário utilizando fone de ouvido exposto à uma interferência que varia com o tempo, ou seja, ruídos provenientes de diversas fontes como, por exemplo: ruído ambiente (som do vento, chuva ou outros sons da natureza); ou sons antropogênicos, tais como acionamento e operação de máquinas, tráfego de carros, motos e caminhões, entre outros. Essas interferências podem incidir por períodos muito curtos de tempo até casos em que podem ser consideradas estacionárias.

O desenvolvimento da eletrônica embarcada e do algoritmo Fuzzy embarcado são descritos nas subseções a seguir:

\subsection{Eletrônica embarcada para o desenvolvimento do CAV}

Para o desenvolvimento do CAV foi utilizado o microcontrolador STM32F407VG, que possui um núcleo baseado no microprocessador ARM Cortex-M4 de 32 bits, além de blocos de processamento aritmético FPU (Floating Point Unit) para operações com ponto flutuante e instruções SIMD (Single Instruction, Multiple Data) e MAC (Multiply-Accumulate operation) que possibilitam ao microprocessador ser utilizado em aplicações de processamento de sinal digital em tempo real [39]. Outras características do microcontrolador são: memória flash de 1 MByte, memória RAM (Random Access Memory) de 192 kBytes e clock com frequência de até $168 \mathrm{MHz}$.

O projeto foi desenvolvido a partir do kit de desenvolvimento STM32F4 Discovery que além do circuito integrado do microcontrolador possui outros periféricos, como por exemplo: sensor de áudio microfone digital omni-direcional (MP45DT02) e tecnologia MEMS (Micro-ElectroMechanical Systems), CODEC (Coder-Decoder) de áudio com amplificador do tipo classe D integrado (CS43L22), e conexão USB (Universal Serial Bus) OTG (On-The-Go) que possibilita o gerenciamento de outros dispositivos, tais como pen-drives ou outras formas de armazenamento de dados.

Para o desenvolvimento dos algoritmos e codificação foi utilizada a versão gratuita e limitada do ambiente de desenvolvimento integrado Keil MDK-ARM (Microcontroller Development Kit for $A R M)$ que possui ferramentas de compilação, depuração e simulação. A programação foi realizada utilizando linguagem ANSI C.

\subsection{Biblioteca Fuzzy embarcada}

A biblioteca Fuzzy desenvolvida e adaptada a partir do código Embedded Fuzzy Logic v0.30 [36], que implementa computacionalmente os conceitos da teoria de conjuntos Fuzzy na linguagem ANSI C. Esse código embarcado descreve o algoritmo Fuzzy a partir do sistema inferência de Mamdani [36].

As especificações dos conjuntos de fuzzyficação, defuzzyficação e regras são definidas pelos arquivos "user_param.h" e "use_rules.h", respectivamente. Essa biblioteca Fuzzy é composta por 3 arquivos em extensão *.c que são: "defuzz.c", "f_operators.c" e "map_function.c" e 3 arqui- 
vos de cabeçalho (extensão *.h) que são: "pre_struct.h", "user_param.h" e "user_rules.h". As características e funções de cada arquivo no processo computacional de raciocínio Fuzzy estão descritos na Tabela 1.

Tabela 1: Descrição dos arquivos da biblioteca Fuzzy embarcada.

\begin{tabular}{|c|c|}
\hline Arquivos & Descrição \\
\hline pre_struct.h & $\begin{array}{l}\text { Fornece a estrutura principal de diferentes variáveis, contendo as } \\
\text { definições de estruturas (struct) e declarações das funções utilizadas no } \\
\text { programa. }\end{array}$ \\
\hline user_param.h & $\begin{array}{l}\text { Contém os parâmetros atribuídos que definem as funções de } \\
\text { pertinência, faixa dinâmica e o universo de discurso dos conjuntos } \\
\text { Fuzzy de entrada e saída. }\end{array}$ \\
\hline user_rules.h & $\begin{array}{l}\text { Descreve as regras Fuzzy definidas pelo usuário. Neste arquivo são } \\
\text { definidas as regras contendo operações entre conjuntos de intersecção } \\
\text { e união. }\end{array}$ \\
\hline f_operators.c & $\begin{array}{l}\text { Contém o código de execução das relações e operações Fuzzy como } \\
\text { intersecção (T-Norma), união (T-Conorma) e complemento. }\end{array}$ \\
\hline map_function.c & Contém o código de execução do mapeamento dos conjuntos Fuzzy. \\
\hline defuzz.c & $\begin{array}{l}\text { Contém o código de execução da defuzzyficação utilizando o método } \\
\text { do centroide. }\end{array}$ \\
\hline
\end{tabular}

A implementação dos arquivos que compõem a biblioteca Fuzzy ocupou espaço em memória, após compilação, de 10.972 Bytes, valor muito inferior comparado com a capacidade máxima da memória flash de 1 MBytes. A biblioteca Fuzzy, composta de funções complexas, requer uma condição computacional crítica quando desenvolvida para soluções embarcadas exigindo, de modo geral, alta capacidade de processamento para gerar resultados em tempo real. Analisando os dados obtidos da compilação desta biblioteca escrita em linguagem ANSI C, verifica-se que sua aplicação é viabilizada sem limitações, ainda que a capacidade de processamento da placa de desenvolvimento (memória flash 1 MBytes) seja elevada. Por ser uma biblioteca leve (lightweight library) todo o código foi implementado utilizando alocação estática. Com relação ao tamanho de dados gerados na compilação e a linguagem de desenvolvimento da biblioteca, torna-se viável a manipulação e implementação do código em diversas outras plataformas e dispositivos embarcados. Além disso, a forma com que a biblioteca foi desenvolvida tem caráter flexível do ponto de vista da adaptação do código para qualquer outro tipo de sistema Fuzzy desenvolvido.

\subsection{Sistema de CAV: aquisição e tratamento do sinal via sensor de áudio - microfone, lógica Fuzzy e biblioteca de reprodução de áudio}

A aplicação do CAV no microcontrolador está detalhada no diagrama em blocos da Figura 3. O sistema utiliza a interface USB OTG para a reprodução sonora armazenada em arquivos de áudio 
com extensão WAV. As faixas utilizadas têm o formato de áudio PCM (Pulse-code modulation), com taxa de amostragem de $44100 \mathrm{kSa} / \mathrm{s}, 16$ bits por amostra e 2 canais estéreo. Os processos de filtragem e gravação são gerenciados utilizando um buffer interno duplo através de filtros digital implementados na biblioteca PDM (Pulse-Density Modulation).

Ao mesmo tempo em que é reproduzido o arquivo de áudio no fone de ouvido do usuário, o CODEC amostra sinais do canal de microfone (monofônico), cujos dados são modulados por duração de pulsos (PDM) que na sequência são convertidos em formato de modulação por código de pulsos (PCM) de 16 bits com frequência de amostragem de $16 \mathrm{kSa} / \mathrm{s}$. A amostragem é realizada utilizando uma janela de 3 segundos e as amostras são armazenadas em um buffer circular para otimizar o desempenho do microprocessador durante o processamento dos dados.

Para realizar a extração de características do sinal de ruído externo, podem ser aplicados diferentes algoritmos [19,35,42]. O método mais simples pode ser feito utilizando limiares de amplitude (thresholds), que indicam diferentes níveis de ruído. Esse método, embora possua rápida resposta para ruídos transitórios, é ineficiente para ruídos estacionários. Dessa forma, podem ser utilizadas técnicas que caracterizam o nível de ruído baseadas no comportamento de uma série de dados amostrados e calculados através de diferentes definições matemáticas, tais como valor RMS (Root Mean Square), SSI (Simple Square Integral), LOG (Log Detector), entre outras [42]. Esses valores são calculados e comparados com níveis de ruídos pré-calibrados. Para o trabalho em questão, optou-se pelo uso da extração via MAV (Mean Absolute Value) por apresentar pouco custo computacional e bom desempenho na indicação de nível de ruído, tal como descrito em (3.1):

$$
M A V=\frac{1}{N} \sum_{n=1}^{N}\left|x_{n}\right|
$$

no qual MAV é a média aritmética do módulo de todas as medidas, $x_{n}$ são os valores das amostras de ruído e $N$ representa o número total de amostras armazenadas no buffer.

O valor da MAV foi utilizado como entrada para o sistema Fuzzy que controla o volume sonoro da saída de áudio estéreo gerada no fone de ouvido. Ou seja, a partir da saída da lógica Fuzzy, fazse o controle do volume que pode variar entre 0 e 100. Os parâmetros de conforto relacionados ao volume e seus limites foram determinados experimentalmente. Valores de volume menores que 45 eram inaudíveis e valores de volume acima de 80 são incômodos e desconfortáveis em um fone de ouvido a ponto do usuário não suportar por muito tempo à exposição sonora. Para os valores de volume acima de 80 até 100, estima-se nível de pressão sonora maiores do que 100 $\mathrm{dB}$ podendo alcançar até $115 \mathrm{~dB}$, considerando um fone de ouvido com sensibilidade de $105 \mathrm{~dB}$ $\mathrm{SPL} / \mathrm{mW}$. Esta faixa de valores está acima dos níveis indicados de no máximo 85 dB [7,10], sendo danosos à audição. Além disso, no experimento, à medida que o usuário foi exposto à um nível de sinal de ruído, determinou-se um valor correspondente de volume confortável de modo a não interferir na inteligibilidade do som reproduzido, ainda que exposto à uma condição mínima de ruído externo. Com base nisso, determinou-se limitar a escala de volume controlado para que a resposta devolvida ao usuário fosse íntegra e consistente. 


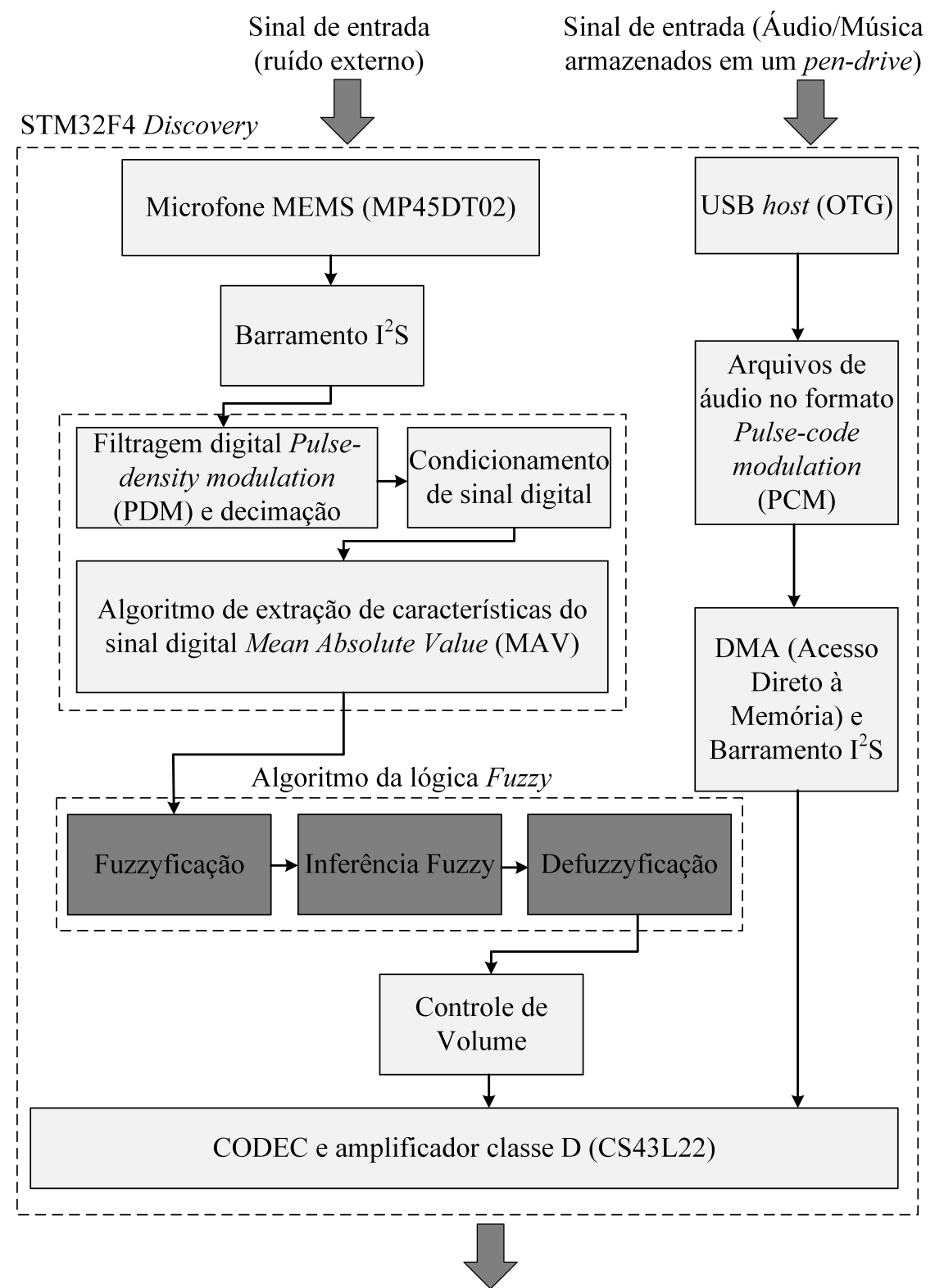

Sinal de saída em um fone de ouvido (Áudio/Música com ajuste de volume)

Figura 3: Diagrama de blocos do CAV baseado em lógica Fuzzy e implementado no microcontrolador ARM Cortex M4. 
Para caracterização dos níveis de ruído e o adequado controle de volume, foram levados em consideração aspectos psicoacústicos da capacidade de discriminação de áudios do tipo discursivos (desde áudio livros até músicas) [20]. O ruído imposto ao microfone foi gerado por uma caixa de som com potência de $3 \mathrm{~W}$ a 0,5 metros de distância do microfone, reproduzindo um ruído, variando-se o volume de 0 a 100 e verificando o valor resultante no buffer PCM, especificamente, através do valor do atributo extraído MAV e também através de um decibelímetro. Todo procedimento de coleta das amostras foi realizado em ambiente acústico (estúdio de música) a fim de garantir a captação de áudio da caixa de som sem a influência de outros sons que pudessem variar a amplitude das amostras.

Com base na comparação entre o ruído externo e o volume necessário para nítida reprodução de áudio no fone de ouvido, foram desenvolvidos os conjuntos Fuzzy de entrada e saída, bem como regras de inferência que permitiram o controle adequado de volume em tempo real.

\section{RESULTADOS E DISCUSSÕES}

\subsection{Integração com biblioteca de reprodução e gravação de áudio e descrição do sistema de inferência Fuzzy}

Os valores amostrados a partir de diferentes níveis de ruído são descritos por valores entre 0 e 65535, que corresponde aos dados não tratados fornecidos pelo conversor analógico-digital de 16 bits, ou seja, entre 0 e $2^{16}-1$. A relação com uma medida conhecida de intensidade sonora foi caracterizada, posteriormente, dentro de uma magnitude pertinente à aplicação utilizando um decibelímetro. Para o modelamento do sistema Fuzzy embarcado no CAV da saída de áudio estéreo, considerou-se a infraestrutura da eletrônica apresentada na Figura 4, analisando o comportamento das variáveis, volume do ruído emitido pela fonte externa e faixa de valores de ruído amostrados pelo microcontrolador em diferentes condições com auxílio da ferramenta de depuração do software de desenvolvimento. Os valores de intensidade sonora do ruído foram variados em diferentes níveis, em conjunto foram comparados com os valores de ruído reconhecidos pelo sistema embarcado e o volume necessário para que fosse possível escutar com nitidez o som reproduzido a partir da memória (áudio e música armazenados no pen-drive). Esses valores serviram de base para o modelamento dos conjuntos Fuzzy de entrada e de saída.

O menor valor de ruído obtido no procedimento foi de, aproximadamente, 5000, na condição de silêncio total sem a reprodução de qualquer som no ambiente em questão. Valores de ruídos próximos a 11000 indicaram um ambiente altamente ruidoso, onde nem mesmo o máximo volume para controle do áudio oriundo da memória foi capaz de melhorar a audição e a compreensão do som gerado.

Para o desenvolvimento do controlador baseado em lógica Fuzzy foram utilizadas: uma variável de entrada, uma variável de saída e um sistema de inferência composto por 5 regras. Os cálculos foram realizados através da composição max-min, sendo o operador min utilizado para o conectivo E nas regras de inferência e o operador max para a agregação da saída. A defuzzyficação foi baseada no método do centroide, tal como descrito anteriormente. 


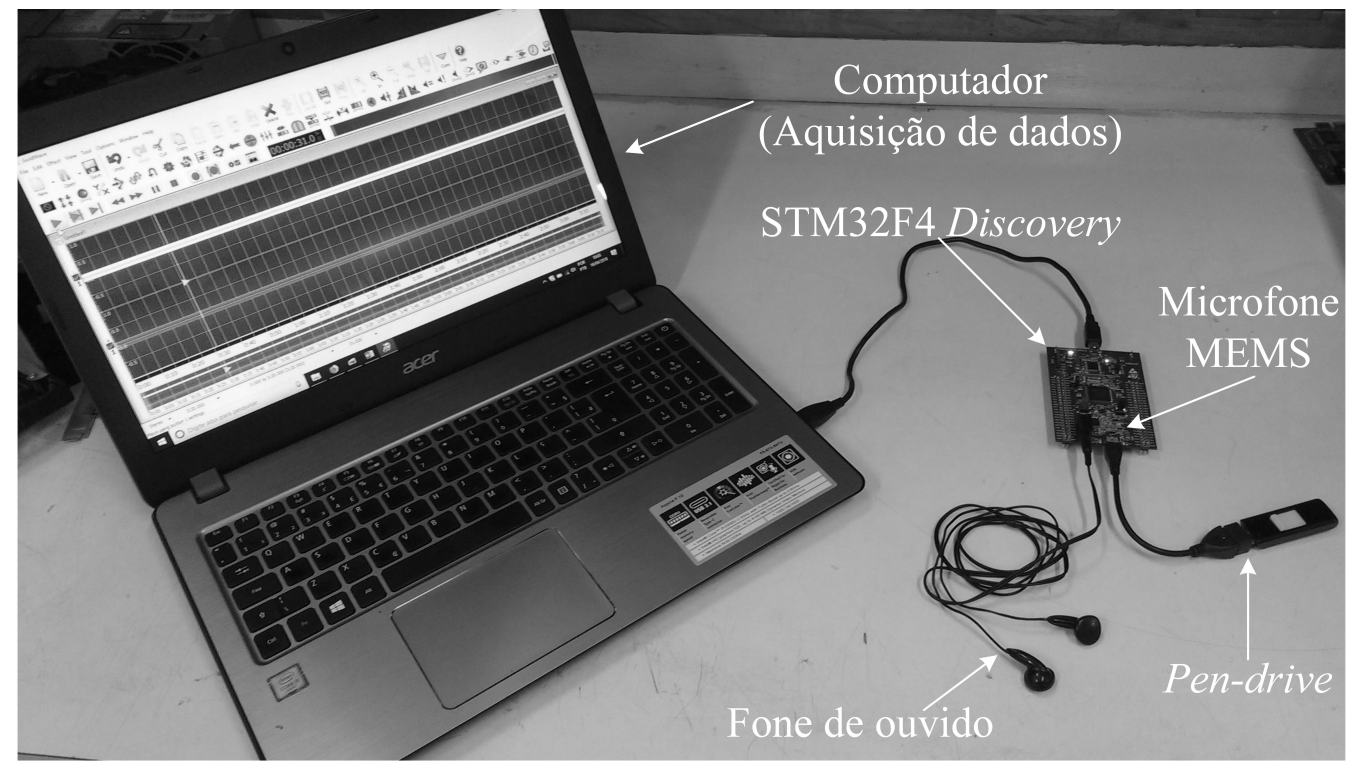

Figura 4: Eletrônica embarcada e esquema de conexões para execução do CAV a partir de inferência Fuzzy.

Com base na análise de correlação entre volume e ruído, desenvolveram-se os conjuntos Fuzzy, no qual o conjunto de entrada característico da variável linguística Nivel de ruído externo é representada por 5 termos linguísticos, sendo eles: baixo (B), médio baixo (MB), médio (M), médio alto (MA) e alto (A). Esses termos são descritos por funções de pertinências trapezoidais e triangulares com uma sobreposição de, aproximadamente, $50 \%$ entre funções.

Em geral, para todos os ensaios realizados, a faixa dinâmica da entrada ficou dentro do intervalo de valores de 3000 a 11000. Para mapear todas os possíveis valores de entradas, o domínio do universo de discurso dos conjuntos Fuzzy de entrada foi descrito no intervalo de [0, 65535]. Para o controlador Fuzzy, o maior detalhamento do universo do discurso de entrada ocorre para valores entre 6000 a 10000, que corresponde aos típicos valores analisados neste trabalho. Todos os valores que sejam menores do que 3000 ou maiores do que 11000, são identificados pelos termos linguísticos B e A, que correspondem aos valores de mínimo e máximo valor de entrada, respectivamente.

Da mesma forma, o conjunto Fuzzy de saída característico da variável linguística Volume foi representado por 4 termos linguísticos, sendo eles: baixo (B), médio baixo (MB), médio alto (MA) e alto (A). Esses termos também foram descritos por funções de pertinências trapezoidais e triangulares, com sobreposição também dentro das recomendações, cujo domínio do universo de discurso do conjunto de saída corresponde ao intervalo de [45, 85].

As proposições Fuzzy, para o sistema embarcado em questão, foram desenvolvidas a fim de assimilar os aspectos e as características dos seres humanos durante a audição no ambiente rui- 
doso anteriormente caracterizado. Para visualização das funções de pertinência, dos conjuntos Fuzzy, das regras Fuzzy e para auxílio em testes preliminares, implementou-se o sistema Fuzzy no software matemático MATLAB, utilizando-se o toolbox Fuzzy Logic Designer.

As Figuras 5 e 6 apresentam os conjuntos Fuzzy de entrada (Nível de ruído externo) e saída (Volume) que foram projetadas tanto no MATLAB como no sistema embarcado. A Tabela 2 apresenta as regras Fuzzy desenvolvidas para este sistema.

Tabela 2: Regras Fuzzy para inferência do sistema.

\begin{tabular}{ll}
\hline & \multicolumn{1}{c}{ Regras } \\
\hline 1 & Se (ruído é B) então (volume é B) \\
\hline 2 & Se (ruído é MB) então (volume é B) \\
\hline 3 & Se (ruído é M) então (volume é MB) \\
\hline 4 & Se (ruído é MA) então (volume é MA) \\
\hline 5 & Se (ruído é A) então (volume é A) \\
\hline
\end{tabular}

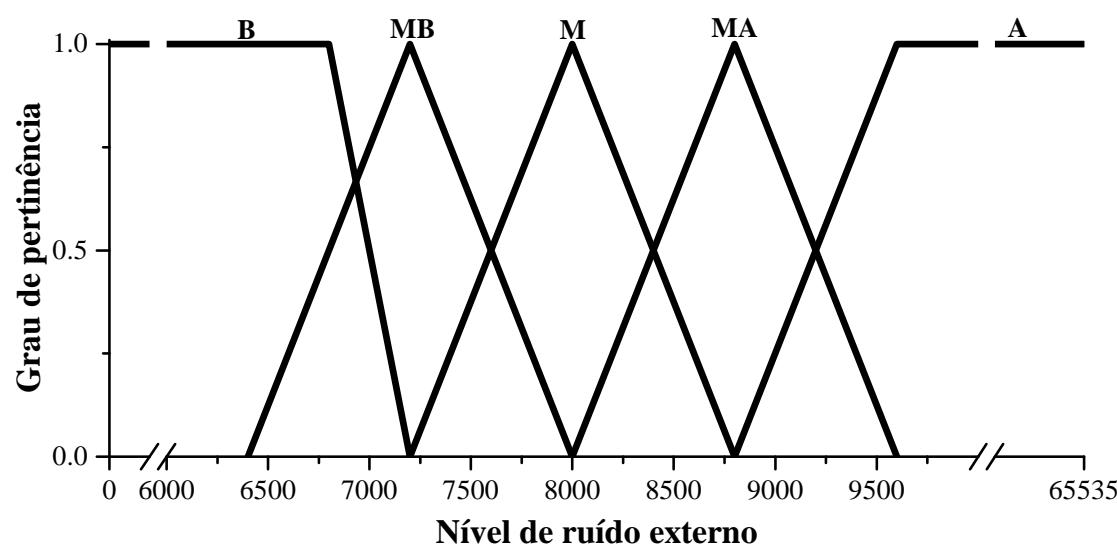

Figura 5: Conjuntos Fuzzy de entrada do Nível de Ruído Externo.

Para medir os tempos de execução do sistema CAV, é possível descrever o diagrama de processamento do CAV em três blocos principais, sendo o primeiro relativo à aquisição dos sinais de ruídos externos pelo microfone, submetendo a amostra coletada ao seu respectivo condicionamento, o qual é composto pelas etapas de filtragem digital (PDM), decimação e extração de caraterísticas. O segundo bloco está relacionado às etapas do algoritmo da lógica Fuzzy: fuzzyficação, inferência Fuzzy e defuzzificação. Por último, o terceiro bloco está relacionado à atuação do sistema através do CAV e à saída de áudio realizada CODEC. Após medir o número de ciclos de clock executados em cada bloco e considerando o microprocessador operando na frequência de clock de $168 \mathrm{MHz}$, verificou-se que o tempo de execução do primeiro bloco é de $27,44 \mathrm{~ms}$, do segundo bloco é de $2,53 \mathrm{~ms}$ e do terceiro bloco é de $0,92 \mathrm{~ms}$. Ou seja, a realização de uma rotina de controle CAV possui tempo total de processamento de $30,89 \mathrm{~ms}$. 


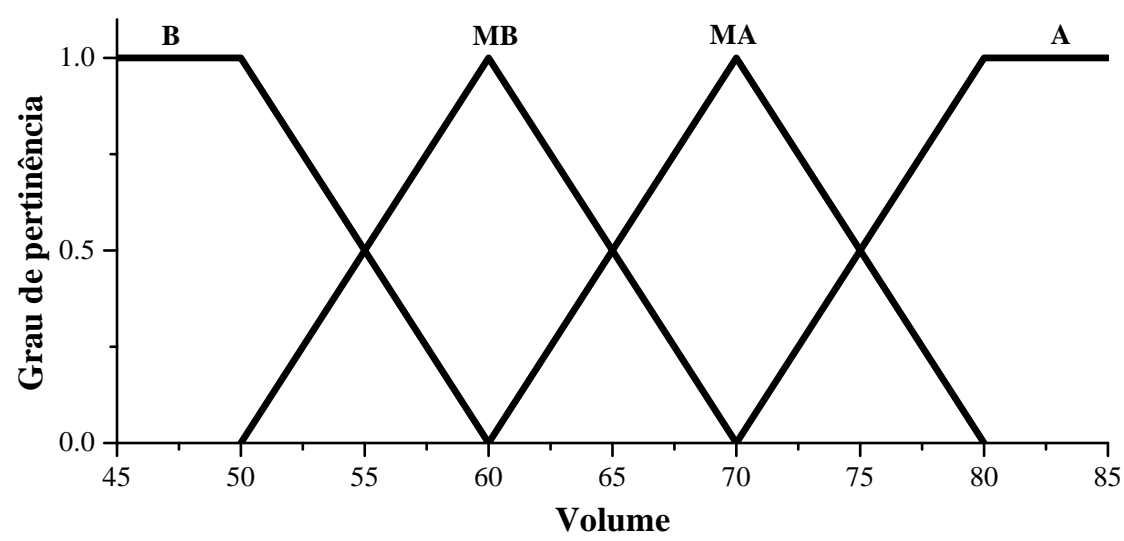

Figura 6: Conjuntos Fuzzy de saída através do ajuste de Volume.

Especificamente em relação ao algoritmo Fuzzy foram necessários 424.227 ciclos de clock para a realização de uma rotina de ação de controle. Sendo 3643 ciclos de clock para a fuzzyficação, 9650 ciclos de clock para o processo de inferência e 410984 ciclos de clock para a defuzzyficação. Em termos da métrica Fuzzy Logic Inference per Second (FLIPS), o sistema embarcado é capaz de realizar, aproximadamente, 156.683 FLIPS.

\subsection{Caracterização do Controlador Automático de Volume Fuzzy}

Após a implementação da lógica Fuzzy embarcada foram avaliados o comportamento do sistema e os resultados de volume em diversas condições. Os testes do CAV foram realizados baseando-se na composição apresentada na Figura 4. Foi proposto um mesmo sinal de ruído, um tom senoidal de $1 \mathrm{kHz}$, variando-se sua amplitude.

A fonte emissora de ruído e as condições dos testes foram mantidas em relação ao procedimento de coleta dos dados para o desenvolvimento dos conjuntos Fuzzy. O áudio de saída com volume controlado foi uma faixa original de uma música previamente armazenada no pen-drive. Através da ferramenta de depuração do software de programação do kit STM32F4 Discovery foi possível coletar os valores de saída do controlador, no caso o volume proveniente da defuzzyficação do sistema para cada amplitude do sinal de ruído imposto ao microfone. Além disso, foi armazenado o valor do nível de ruído, relacionado a cada amplitude. Esses valores estão apresentados na Figura 7, que descreve o comportamento do CAV para níveis de ruído variando de 5787 a 10489 (aproximadamente entre $21 \mathrm{~dB}$ a $72 \mathrm{~dB}$ ) [28]. Essa figura apresenta de forma gráfica a relação entre o volume do áudio controlado e as variações de intensidade de ruído externo. Observa-se, no eixo das abscissas, os valores de intensidade de ruídos proveniente do algoritmo proposto e sua equivalência em decibéis. Já no eixo das ordenadas, têm-se os valores de volumes oriundos da inferência Fuzzy. Verifica-se que o sistema Fuzzy apresenta comportamento não linear, o que possibilita a incorporação da percepção humana ao CAV, contemplando aspectos subjetivos durante a audição. 


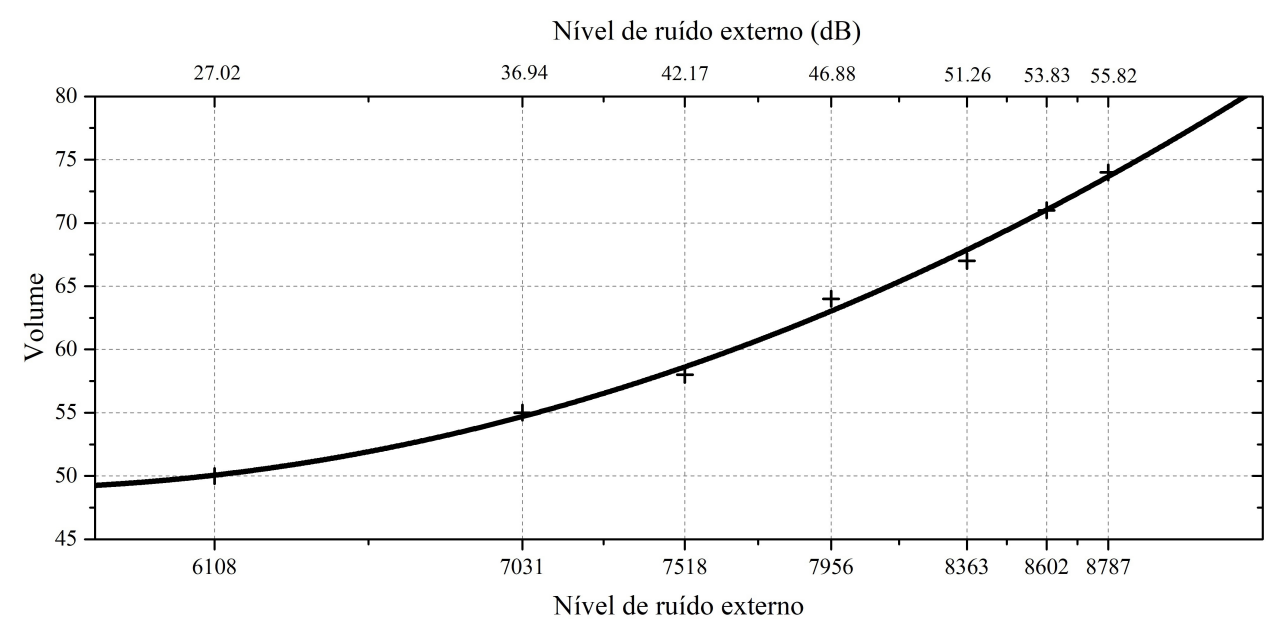

Figura 7: Saída do volume do controlador automático Fuzzy a partir da entrada de diferentes níveis de ruído externo.

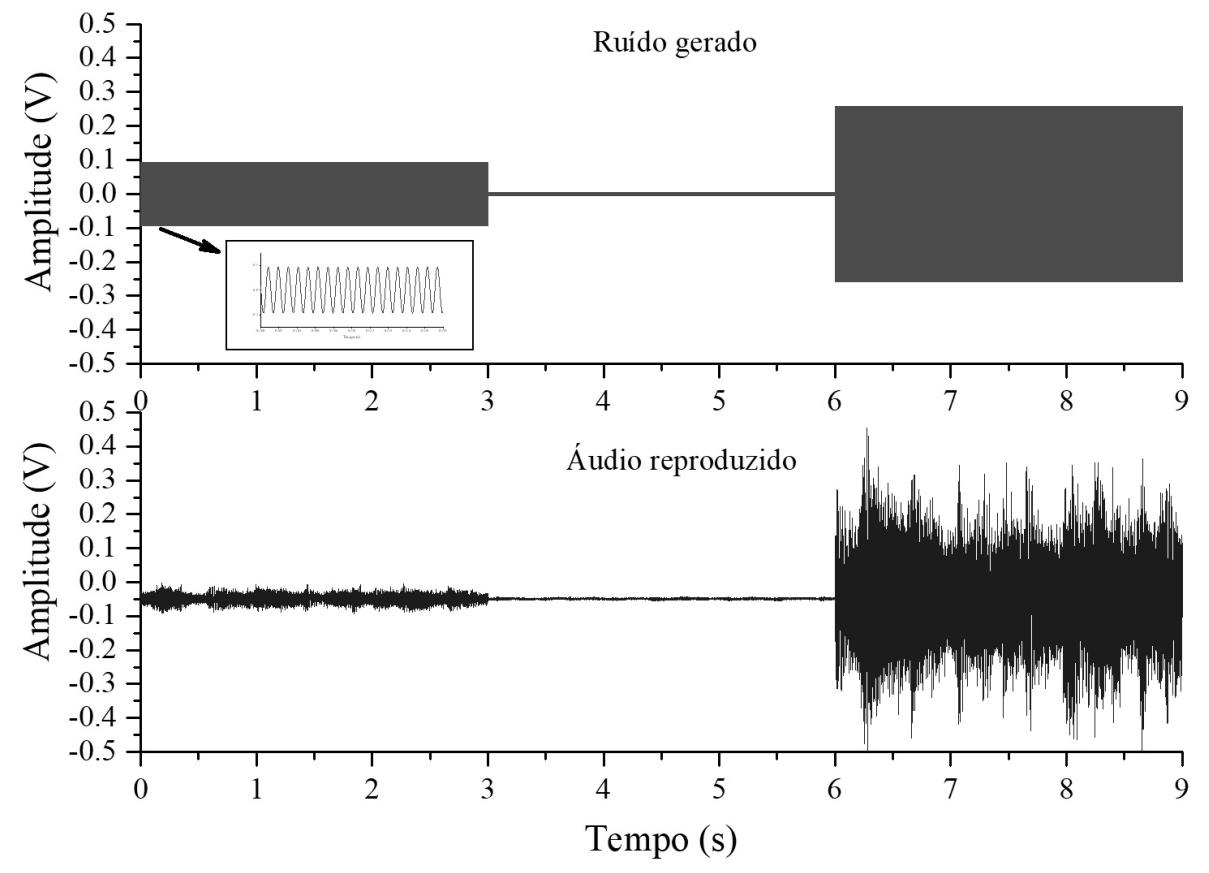

Figura 8: Sinal de ruído nas condições de médio, baixo e alto. A saída do áudio é ajustada a partir da inferência Fuzzy. 
Para visualização do comportamento do sinal de áudio com volume controlado de acordo com a amplitude do ruído, foram captados os sinais de ruído com diferentes amplitudes em 3 condições: ruído médio, ruído baixo e ruído alto, e os sinais da música (saída) provenientes das condições impostas ao controle de volume, conforme descrito na Figura 7.

A capacidade de percepção da fala e a inelegibilidade sonora dependem de vários elementos auditivos e extra-auditivos [20,30]. Em um contexto geral, a relação sinal-ruído, Signal-Noise Ratio (SNR), definida como a relação entre a potência do som desejado e a potência do ruído de fundo, corresponde ao parâmetro mais efetivo para a garantir compreensão e inteligibilidade da audição [20,23]. Em geral, valores maiores do que 3 e 4 dB de SNR garantem percepção sonora adequada $[23,30]$. Para todos os testes realizados, foi possível obter valores maiores do que $5 \mathrm{~dB}$ de SNR.

Verifica-se que o sistema é capaz de responder às variações de intensidade de ruído. Nas três condições de sinal do ruído exemplificadas como entrada, o controlador Fuzzy foi capaz de selecionar um volume de saída automaticamente. Esse controle pode ser visto na amplitude do áudio reproduzido que compensa um maior nível de ruído com uma maior amplitude do sinal do áudio controlado.

Em comparação com outros trabalhos, os resultados obtidos com o controlador Fuzzy são representativos. Felber [9] apresenta um controlador que garante valor mínimo de ganho de 2,5 dB do sinal desejado em relação ao ruído de entrada. Enquanto, Takahashi et al. [35] apresenta como resultados questionários qualitativos para avaliar a percepção sonora de dois participantes na interação com um robô dentro de um ambiente ruídoso, com emissão de ruído ambiente de $55 \mathrm{~dB}$. Haymizu et al. [14] também apresenta resultados qualitativos obtidos por questionários de 10 participantes. De modo semelhante, Yang et al. [38] analisa seus resultados baseados na taxa de reconhecimento de palavras.

Em relação ao tempo de execução, resultados semelhantes podem ser encontrados no trabalho de Roza e Fernandes [29]. Nele, uma das métricas avaliadas para a validação da plataforma proposta para gerenciamento de máquinas de inferência Fuzzy foi o tempo de processamento para várias quantidades de regras Fuzzy. Para uma máquina de inferência Fuzzy concebida com 9 regras, o tempo de execução foi de $0,20 \mathrm{~ms}$ considerando um microprocessador com frequência de clock de $400 \mathrm{MHz}$. Em comparação com o tempo de 2,53 ms obtidos no microprocessador com frequência de clock de $168 \mathrm{MHz}$, fica claro que a plataforma aqui apresentada é uma alternativa viável para a implementação de controladores Fuzzy, satisfazendo as necessidades operacionais de diversas aplicações de sistemas de controle, garantido baixa latência e alta capacidade de vazão média de dados (throughput).

\section{CONCLUSÕES}

Foi implementado um CAV baseado em lógica Fuzzy, permitindo aproximar o raciocínio humano e aspectos psicoacústicos na tomada de decisão do controlador. Além disso, outras características da lógica Fuzzy ficaram evidentes no sistema concebido, entre elas: a forma com que a lógica 
Fuzzy simplifica a solução do problema; a aproximação do raciocínio humano que o uso de variáveis linguísticas proporcionam; e a maior capacidade de intervenção do usuário em relação ao controle do sistema com o uso de valores de pertinências.

O presente trabalho descreveu uma metodologia de implementação de uma estrutura abrangente para a implementação da lógica Fuzzy operando em tempo real e embarcada em um sistema portátil. Foram adaptadas e embarcadas todas as bibliotecas necessárias para se realizar a lógica Fuzzy em um microcontrolador ARM Cortex M4 de baixo custo e alta capacidade de processamento. Estas bibliotecas foram detalhadas para possilitar a rápida prototipação de controladores Fuzzy embarcados. Além disso foram apresentados os resultados da caracterização de tempo de processamento do controlador Fuzzy e memória necessário para implementação da codificação. As bibliotecas atenderam à demanda de espaço em memória e processamento, principalmente a biblioteca Fuzzy por apresentar uma situação computacional crítica quando utilizada em aplicações de tempo real em uma solução embarcada. Os resultados indicam que a biblioteca é viável para diferentes aplicações de sistemas de controle. Além disso, a biblioteca Fuzzy pode ser aplicada sem limitação de tamanho e de tempo de processamento dentro dos microprocessadores equivalentes ao utilizado neste trabalho.

O modelo Fuzzy proposto para realizar o CAV a partir do ruído ambiente mostrou-se eficiente para atender a aplicação proposta. $\mathrm{O}$ modelo foi concebido avaliando-se de forma prática e subjetiva a capacidade de discriminação de sons em um ambiente ruídos, com objetivo de tornar o áudio controlado confortável e adaptado às características humanas. Possibilitando o ajuste de volume para ruídos variando entre $21 \mathrm{~dB}$ e $72 \mathrm{~dB}$. Garantindo o ajuste do nível sonoro até os limiares de no máximo $100 \mathrm{~dB}$. Na prática, as músicas as quais o volume estava sendo controlado puderam ser escutadas nitidamente sem que o ruído externo prevalecesse. Mesmo durante a audição de músicas com intensidade sonora menor, tal como músicas clássicas e áudio livros, o ruído não prejudicou a inteligibilidade do som.

Todos os testes e caracterizações foram realizadas em diversas condições, considerando ruídos em amplitudes diferentes e analisando-se o comportamento da variável de saída do controlador Fuzzy. O CAV utilizando a biblioteca Fuzzy demonstrou a potencialidade da aplicação de algoritmos inteligentes embarcados em sistemas de controle e sua potencialidade para ser implementado em outras aplicações de controle.

\footnotetext{
ABSTRACT. Automatic Volume Control (AVC) is a technology that automatically adjusts the output power of the speaker in the presence of noise. Different techniques to perform the AVC have been reported in the literature. However, only a few studies use computational intelligence algorithms. Fuzzy logic is an computational intelligence technique that supports reasoning modes with the uncertainty principle. The Fuzzy algorithm can approximate human thinking and psychoacoustic aspects in audio applications. This paper presents an AVC based on the Fuzzy logic embedded algorithm. The proposed system allows realtime volume control of an audio signal in a noisy environment. The Fuzzy algorithm was implemented in ANSI C language and embedded in an ARM Cortex M4 microprocessor. The results demonstrated the automatic and fast adjustment of the output audio volume th-
} 
rough Fuzzy rules, confirming the potential of Fuzzy logic in audio applications, in control applications as well as in the decision making of critical systems.

Keywords: fuzzy logic, multimedia systems, embedded systems, Automatic Volume Control.

\section{REFERÊNCIAS}

[1] O. Al-Jarrah \& A. Shaout. Automotive Volume Control Using Fuzzy Logic. J. Intell. Fuzzy Syst., 18(4) (2007), 329-343. URL http://dl . acm . org/citation. cfm?id=1368416.1368417.

[2] C.K. Barker et al. Automatic volume control for land mobile radio (31 July 2014). U. S. Patent 20180205354A1.

[3] A.M. Bertone, R. Jafelice \& M. Câmara. Fuzzy Linear Programming: Optimization of an Electric Circuit Model. Trends in Applied and Computational Mathematics, 18(3) (2018), 419. doi:10.5540/ tema.2017.018.03.419. URL https://tema.sbmac.org.br/tema/article/view/989.

[4] A.M. Bertone, J. Martins \& K. Yamanaka. Black-Box Fuzzy Identification of a Nonlinear Hydrogen Fuel Cell Model. Trends in Applied and Computational Mathematics, 18(3) (2018), 405. doi:10.5540/ tema.2017.018.03.405. URL https://tema.sbmac.org.br/tema/article/view/968.

[5] A. Bressane, F. Fengler, S. Roveda, J. Roveda \& A. Martins. Arboreal Identification Supported by Fuzzy Modeling for Trunk Texture Recognition. Trends in Applied and Computational Mathematics, 19(1) (2018), 111. doi:10.5540/tema.2018.019.01.111. URL https : //tema . sbmac . org. br/tema/ article/view/1079.

[6] S.M. Cassidy, L.E. Moravetz \& M.J. Dietel. Speaker with Automatic Volume Control (08 January 2018). U. S. Patent US20190214957A1.

[7] I. dos Santos, M.F. Colella-Santos \& C.M. do Couto. Pressão sonora gerada por equipamentos sonoros portáteis individuais. Brazilian Journal of Otorhinolaryngology, 80 (2014), 41 - 47.

[8] M. Fayaz \& D.H. Kim. An Efficient Actuator Control Mechanism using Fuzzy Logic on Embedded System. International Journal of Control and Automation, 11(6) (2018), 35-44. doi:http//dx.doi.org/ 10.14257/ijca.2018.11.6.04.

[9] F. Felber. An automatic volume control for preserving intelligibility. In "34th IEEE Sarnoff Symposium" (2011), pp. 1-5.

[10] B.J. Fligor \& L.C. Cox. Output Levels of Commercially Available Portable Compact Disc Players and the Potential Risk to Hearing. Ear and Hearing, 25(6) (2004), 513-527.

[11] C. Gómez, M. Vellasco \& R. Tanscheit. Controle de um Sistema de Navegação de um Robô Ambiental Híbrido por meio de um Sistema de Inferência Fuzzy Hierárquico. Trends in Applied and Computational Mathematics, 19(2) (2018), 235. doi:10.5540/tema.2018.019.02.235. URL https: //tema.sbmac.org.br/tema/article/view/1100.

[12] B. Griffith \& J. Tom. Audio automatic volume control circuit. In "1956 IEEE International Solid-State Circuits Conference. Digest of Technical Papers” (1956), pp. 3-3. 
[13] K.P. Han, K.W. Song, Z.H. Kim, G.C. Lee \& Y.H. Ha. Automatic volume control system for compensation of volume difference between TV channels. IEEE Transactions on Consumer Electronics, 43(4) (1997), 1197-1205. doi:10.1109/30.642387.

[14] A. Hayamizu, M. Imai, K. Nakamura \& K. Nakadai. Volume Adaptation and Visualization by Modeling the Volume Level in Noisy Environments for Telepresence System. In "Proceedings of the Second International Conference on Human-agent Interaction", HAI '14. ACM, New York, NY, USA (2014), pp. 67-74. doi:10.1145/2658861.2658875. URL http://doi.acm.org/10.1145/ 2658861.2658875.

[15] Y. Hwangbo, C.J. Park \& S.H. Choi. Automatic Volume Control Based on Background Noise Characteristics. International Journal on Recent and Innovation Trends in Computing and Communication (IJRITCC), 4(12) (2016), 40-41.

[16] M. Iphar \& R. Goktan. An application of fuzzy sets to the Diggability Index Rating Method for surface mine equipment selection. International Journal of Rock Mechanics and Mining Sciences, 43(2) (2006), 253 - 266. doi:https://doi.org/10.1016/j.jirmms.2005.07.003. URL http: //www . sciencedirect.com/science/article/pii/S1365160905001103.

[17] H.M.D. Kabir, M.E. Rahman, A.Z. Hassan \& M.N. Uddin. Correlation Based Automatic Volume Control System for Television/ Radio. International Journal of Computing and Digital Systems, 3(2) (2014), 151-160. doi:http://dx.doi.org/10.12785/IJCDS/030209.

[18] S.G. Kambalimath, P.C. Pandey, P.N. Kulkarni, S.S. Mahant-Shetti \& S.G. Hiremath. FPGA-based Design of a Hearing Aid with Frequency Response Selection through Audio Input. In "2016 29th International Conference on VLSI Design and 2016 15th International Conference on Embedded Systems (VLSID)" (2016), pp. 579-580. doi:10.1109/VLSID.2016.87.

[19] F.S. Kinsler et al. Automatic volume control to compensate for speech interference noise. U. S. Patent 7,760,893 (20 July 2010), U. S. Patent 7,908,134 (15 March 2011).

[20] L. Kinsler, A. Frey, A. Coppens \& J. Sanders. "Fundamentals of Acoustics". Wiley, 4 ed. (2000).

[21] R. Kulavik, S.D.K. Buchannagari \& C. Bonanno. Automatic volume control for combined game and chat audio (18 August 2018). U. S. Patent US10236849B2.

[22] E. Li. Automatic volume control based on context and location (03 July 2013). U. S. Patent 20150011195A1.

[23] P.C. Loizou. "Speech Enhancement: Theory and Practice". CRC Press, 2 ed. (2013).

[24] E.H. Mamdani. Application of Fuzzy Logic to Approximate Reasoning Using Linguistic Synthesis. IEEE Transactions on Computers, C-26(12) (1977), 1182-1191. doi:10.1109/TC.1977.1674779.

[25] E.H. Mamdani \& S. Assilian. An Experiment in Linguistic Synthesis with a Fuzzy Logic Controller. Int. J. Hum.-Comput. Stud., 51(2) (1999), 135-147. doi:10.1006/ijhc.1973.0303. URL http://dx. doi.org/10.1006/ijhc.1973.0303.

[26] E. Martinson \& D. Brock. Auditory Perspective Taking. IEEE Transactions on Cybernetics, 43(3) (2013), 957-969. doi:10.1109/TSMCB.2012.2219524. 
[27] G. Oltean \& L. Ivanciu. Implementation of a fuzzy logic-based embedded system for temperature control. In "2017 40th International Spring Seminar on Electronics Technology (ISSE)” (2017), pp. 1-6. doi:10.1109/ISSE.2017.8001006.

[28] M.G.S. Pires et al. Medida do nível de pressão sonora em um centro urbano. J. Bras. Fonoaudiol., 3(13) (2012), 263-266.

[29] S. Roza \& M. Fernandes. OpenFZ: Uma Plataforma Embarcada para Gerenciamento de Máquinas Fuzzy. In "Congresso Brasileiro de Sistemas Fuzzy - V CBSF, Fortaleza, CE, Brazil” (2018).

[30] E. Shojaei, H. Ashayeri, Z. Jafari, M.R. Zarrin Dast \& K. Kamali. Effect of signal to noise ratio on the speech perception ability of older adults. Medical journal of the Islamic Republic of Iran, 30 (2016), 342-342. URL https://www.ncbi.nlm.nih.gov/pubmed/27390712.

[31] M.G. Simões \& I.S. Shaw. “Controle e Modelagem Fuzzy”. São Paulo: Edgard Blucher Ltda (1999).

[32] J. Soto-Hidalgo, A. Vitiello, J. Alonso, G. Acampora \& J. Alcala-Fdez. Design of Fuzzy Controllers for Embedded Systems With JFML. International Journal of Computational Intelligence Systems, 12 (2018), 204-214. doi:https://doi.org/10.2991/ijcis.2019.125905646. URL https://doi. org/10.2991/ijcis.2019.125905646.

[33] D.A. Srail. Automatic volume control based on speech recognition (15 October 2014). U. S. Patent 9508344B2.

[34] M. Sugeno \& G. Kang. Structure identification of fuzzy model. Fuzzy Sets and Systems, 28(1) (1988), 15 - 33. doi:https://doi.org/10.1016/0165-0114(88)90113-3. URL http://www . sciencedirect. com/science/article/pii/0165011488901133.

[35] M. Takahashi, M. Ogata, M. Imai, K. Nakamura \& K. Nakadai. A case study of an automatic volume control interface for a telepresence system. In "2015 24th IEEE International Symposium on Robot and Human Interactive Communication (RO-MAN)" (2015), pp. 517-522. doi:10.1109/ROMAN. 2015.7333605 .

[36] S.H. Teay. Embedded Fuzzy Logic v0.30 (2019). URL https://github.com/Beta-10/ embedded-fuzzy-logic. Accessed: 2019-01-20.

[37] H.A. Wheeler. Automatic Volume Control for Radio Receiving Sets. Proceedings of the Institute of Radio Engineers, 16(1) (1928), 30-34. doi:10.1109/JRPROC.1928.221286.

[38] J. Yang, P. Hilmes, B. Adair \& D.W. Krueger. Deep learning based automatic volume control and limiter system. In "2017 IEEE International Conference on Acoustics, Speech and Signal Processing (ICASSP)" (2017), pp. 2177-2181. doi:10.1109/ICASSP.2017.7952542.

[39] J. Yiu. "The Definitive Guide to ARM Cortex-M3 and Cortex-M4 Processors". Elsevier (2014).

[40] L.A. Zadeh. Fuzzy Sets. Information and Control, 8(3) (1965), 338-353. doi:10.1016/ S0019-9958(65)90241-X. URL https://doi .org/10.1016/S0019-9958(65) 90241-X.

[41] L.A. Zadeh. Fuzzy logic - a personal perspective. Fuzzy Sets and Systems, 281 (2015), 4-20. doi:10. 1016/j.fss.2015.05.009. URL https://doi.org/10.1016/j.fss. 2015.05.009. 
[42] T. Zhang \& J. Kuo. Audio content analysis for online audiovisual data segmentation and classification. IEEE Transactions on Speech and Audio Processing, 9(4) (2001), 441-457. doi:10.1109/89.917689.

(cc) BY 